TRANSACTIONS OF THE

AMERICAN MATHEMATICAL SOCIETY

Volume 361, Number 5, May 2009, Pages 2607-2630

S 0002-9947(08)04601-1

Article electronically published on November 24, 2008

\title{
SUBELLIPTIC ESTIMATES FOR SOME SYSTEMS OF COMPLEX VECTOR FIELDS: QUASIHOMOGENEOUS CASE
}

\author{
M. DERRIDJ AND B. HELFFER
}

\begin{abstract}
For about twenty five years it was a kind of folk theorem that complex vector-fields defined on $\Omega \times \mathbb{R}_{t}$ (with $\Omega$ open set in $\mathbb{R}^{n}$ ) by

$$
L_{j}=\frac{\partial}{\partial t_{j}}+i \frac{\partial \varphi}{\partial t_{j}}(\mathbf{t}) \frac{\partial}{\partial x}, j=1, \ldots, n, \mathbf{t} \in \Omega, x \in \mathbb{R},
$$

with $\varphi$ analytic, were subelliptic as soon as they were hypoelliptic. This was the case when $n=1$, but in the case $n>1$, an inaccurate reading of the proof given by Maire (see also Trèves) of the hypoellipticity of such systems, under the condition that $\varphi$ does not admit any local maximum or minimum (through a nonstandard subelliptic estimate), was supporting the belief for this folk theorem. Quite recently, J.L. Journé and J.M. Trépreau show by examples that there are very simple systems (with polynomial $\varphi$ 's) which are hypoelliptic but not subelliptic in the standard $L^{2}$-sense. So it is natural to analyze this problem of subellipticity which is in some sense intermediate (at least when $\varphi$ is $C^{\infty}$ ) between the maximal hypoellipticity (which was analyzed by Helffer-Nourrigat and Nourrigat) and the simple local hypoellipticity (or local microhypoellipticity) and to start first with the easiest nontrivial examples. The analysis presented here is a continuation of a previous work by the first author and is devoted to the case of quasihomogeneous functions.
\end{abstract}

\section{IntRodUCTION AND MAIN RESUlT}

1.1. Preliminaries. Let $\Omega$ be an open set in $\mathbb{R}^{n}$ with $0 \in \Omega$. We consider the regularity properties of the following family of complex vector fields on $\Omega \times \mathbb{R}$ :

$$
L_{j}=\frac{\partial}{\partial t_{j}}+i \frac{\partial \varphi}{\partial t_{j}}(\mathbf{t}) \frac{\partial}{\partial x}, j=1, \ldots, n, \mathbf{t} \in \Omega, x \in \mathbb{R},
$$

where $\varphi \in C^{1}(\Omega, \mathbb{R})$, with $\varphi(0)=0$. We will concentrate our analysis near a point $(0,0)$ (but note that the operator is invariant by translation in the $x$ variable).

Many authors have considered this type of system. For a given $\Omega$, they were in particular interested in the existence, for some pair $(s, N)$ such that $s+N>0$, of the following family of inequalities.

For any pair of bounded open sets $\omega, I$ such that $\bar{\omega} \subset \Omega$ and $I \subset \mathbb{R}$, there exists a constant $C_{s, N}(\omega, I)$ such that

$$
\|u\|_{s}^{2} \leq C_{N}(\omega, I)\left(\sum_{j=1}^{n}\left\|L_{j} u\right\|_{0}^{2}+\|u\|_{-N}^{2}\right), \forall u \in C_{0}^{\infty}(\omega \times I),
$$

where $\|\cdot\|_{r}$ denotes the Sobolev norm in $H^{r}(\Omega \times \mathbb{R})$.

Received by the editors January 8, 2007 and, in revised form, July 19, 2007.

2000 Mathematics Subject Classification. Primary 35B65; Secondary 32N15.

(C)2008 American Mathematical Society 2607

Reverts to public domain 28 years from publication 
If $s>0$, we say that we have a subelliptic estimate. In [JoTre, there are also results where $s$ can be arbitrarily negative. We will speak of the case when $s \leq 0$ of weak subellipticity. Note that in this case the existence of this inequality is not sufficient for proving hypoellipticity.

The system (1.1) being elliptic in the $\mathbf{t}$ variable, it is enough to analyze the subellipticity microlocally near $\tau=0$, i.e. near $(0,(0, \xi))$ in $(\omega \times I) \times\left(\mathbb{R}^{n+1} \backslash\{0\}\right)$ with $\{\xi>0\}$ or $\{\xi<0\}$.

This leads to the analysis of the existence of two constants $C_{s}^{+}$and $C_{s}^{-}$such that the two following inequalities hold:

$$
\int_{\omega \times \mathbb{R}^{+}} \xi^{2 s}|\widehat{u}(\mathbf{t}, \xi)|^{2} d t d \xi \leq C_{s}^{+} \int_{\omega \times \mathbb{R}^{+}}|\widehat{L u}(\mathbf{t}, \xi)|^{2} d \mathbf{t} d \xi, \forall u \in C_{0}^{\infty}(\omega \times \mathbb{R}),
$$

where $\widehat{u}(\mathbf{t}, \xi)$ is the partial Fourier transform of $u$ with respect to the $x$ variable, and

$$
\int_{\omega \times \mathbb{R}^{-}}|\xi|^{2 s}|\widehat{u}(\mathbf{t}, \xi)|^{2} d \mathbf{t} d \xi \leq C_{s}^{-} \int_{\omega \times \mathbb{R}^{-}}|\widehat{L u}(\mathbf{t}, \xi)|^{2} d \mathbf{t} d \xi, \forall u \in C_{0}^{\infty}(\omega \times \mathbb{R}) .
$$

When (1.3) is satisfied, we will speak of microlocal subellipticity in $\{\xi>0\}$, and similarly when (1.4) is satisfied, we will speak of microlocal subellipticity in $\{\xi<0\}$. Of course, when $s>0$, it is standard that these two inequalities imply (1.2).

We now observe that (1.3) for $\varphi$ is equivalent to (1.4) for $-\varphi$, so it is enough to concentrate our analysis on the first case.

1.2. The main result. In $[\mathrm{De}$, the first author gave a sufficient condition on $\varphi$ for getting (1.2) with $s>0$. In this article, we consider the case of quasihomogeneous functions $\varphi$ on $\mathbb{R}^{2}$ (i.e. $n=2$ ) and we will give a simple condition of subellipticity where $s$ will be related rather simply with the quasihomogeneity of $\varphi$.

These conditions will be expressed for $\varphi$ in $C^{1}$, but note that they become more simple in the analytic case (see Section 7).

More precisely, let $m$ and $\ell$ in $\mathbb{R}^{+}$be such that

$$
\ell \geq 1
$$

and

$$
m \geq 2 \ell .
$$

We make these two assumptions 11 in the whole paper.

We consider in $\mathbb{R}^{2}(t, s)$ as the variables (instead of $\mathbf{t}$ ), and the functions $\varphi \in$ $C^{1}\left(\mathbb{R}^{2}\right)$ will be quasihomogeneous in the following sense:

$$
\varphi\left(\lambda t, \lambda^{\ell} s\right)=\lambda^{m} \varphi(t, s), \forall(t, s, \lambda) \in \mathbb{R}^{2} \times \mathbb{R}^{+} .
$$

According to (1.7), the real function $\varphi$ is determined by its restriction $\widetilde{\varphi}$ to the distorted circle $\mathcal{S}$

$$
\widetilde{\varphi}:=\varphi_{\mid \mathcal{S}}
$$

where $\mathcal{S}$ is defined by

$$
\mathcal{S}=\left\{(t, s) ; t^{2 \ell}+s^{2}=1\right\} .
$$

Our main result is stated under the following assumption on $\widetilde{\varphi}$.

\footnotetext{
${ }^{1}$ With, in addition, the assumption that $\ell$ is rational in the analytic case.
} 
Assumption $1.1(H 2)$. $\quad$ (i) $\widetilde{\varphi}$ is not strictly negative.

(ii) $\widetilde{\varphi}$ cannot have, at any of its zeroes, a local maximum.

(iii) If $\mathcal{S}_{j}^{+}$is a connected component of $\widetilde{\varphi}^{(-1)}(] 0,+\infty[)$, then one can write $\mathcal{S}_{j}^{+}$ as a finite union of arcs satisfying Property 1.2 below.

(iv) If $\mathcal{S}_{j}^{-}$is one connected component of $\widetilde{\varphi}^{(-1)}(]-\infty, 0[)$, then $\tilde{\varphi}$ has a unique minimum in $\mathcal{S}_{j}^{-}$.

(v) There exists $p \geq 1$, such that, if $\theta_{0}$ is a zero of $\widetilde{\varphi}$, then there exists an open $\operatorname{arc} \mathcal{V}_{\theta_{0}}$ containing $\theta_{0}$ in $\mathcal{S}$ and $C_{0}>0$, such that

$$
\left|\widetilde{\varphi}(\theta)-\widetilde{\varphi}\left(\theta^{\prime}\right)\right| \geq \frac{1}{C_{0}}\left|\theta-\theta^{\prime}\right|^{p}, \forall \theta, \theta^{\prime} \in \mathcal{V}_{\theta_{0}},
$$

with $\theta$ and $\theta^{\prime}$ in the same connected component in $\mathcal{V}_{\theta_{0}} \backslash\left\{\theta_{0}\right\}$.

Here in the third item of $(H 2)$, we say an arc $\left[\theta, \theta^{\prime}\right]$ satisfies Property 1.2 if :

Property 1.2. There exists on this arc a point $\widehat{\theta}$ such that:

(a) $\widetilde{\varphi}$ is nondecreasing on the $\operatorname{arc}[\theta, \widehat{\theta}]$ and nonincreasing on the arc $\left[\widehat{\theta}, \theta^{\prime}\right]$. (So the restriction of $\widetilde{\varphi}$ to $\left[\theta, \theta^{\prime}\right]$ has a maximum at $\widehat{\theta}$.)

(b)

$$
\langle\widehat{\theta} \mid \theta\rangle_{\ell} \geq 0 \text { and }\left\langle\widehat{\theta} \mid \theta^{\prime}\right\rangle_{\ell} \geq 0,
$$

where for $\theta=(\alpha, \beta)$ and $\widehat{\theta}=(\widehat{\alpha}, \widehat{\beta})$ in $\mathcal{S} \subset \mathbb{R}^{2}$,

$$
\langle\widehat{\theta} \mid \theta\rangle_{\ell}:=\widehat{\alpha} \alpha|\widehat{\alpha}|^{\ell-1}|\alpha|^{\ell-1}+\widehat{\beta} \beta .
$$

Note here that we could have $\widetilde{\varphi}$ constant on $\mathcal{S}_{\theta, \theta^{\prime}}$ and $\widehat{\theta}=\theta$ or $\theta^{\prime}$. Moreover, item (b) says that the length of the two arcs is sufficiently small, more precisely that the distorted "angles" (see Section [3) associated to $\left[\theta, \theta^{\prime}\right]$ are acute.

We can now state our main theorem:

Theorem 1.3. Let $\varphi \in C^{1}\left(\mathbb{R}^{2}, \mathbb{R}\right)$ satisfying (1.7), with $\ell$ and $m$ satisfying (1.5) and (1.6). Then $L$ is microlocally subelliptic in $\{\xi>0\}$.

Remarks 1.4.

(i) The proof of Theorem 1.3 consists in showing that Assumption (H2) implies the assumption $\left(H_{+}(\alpha)\right)$ with $\alpha=\frac{1}{\max (m, p)}$ introduced in De] and which will be recalled in Section 2 .

(ii) [De] was considering the homogeneous case $\ell=1$ and $m \geq 2$. Here we generalize by considering the quasihomogeneous case, but the sufficient condition given here for getting Assumption 2.1 satisfied will already be an improvement in the homogeneous case.

(iii) The conditions on $\widetilde{\varphi}$ are more restrictive on the $\operatorname{arcs} S_{k}^{-}$.

(iv) If $\varphi$ is analytic and $\ell$ is rational, the statement of the main theorem becomes simpler. (iii) and (v) are indeed automatically satisfied as soon as $\widetilde{\varphi}$ is not identically 0 . Moreover, if we write $\ell=\frac{\ell_{2}}{\ell_{1}}$ (with $\ell_{1}$ and $\ell_{2}$ mutually prime integers), all the criteria involving $\widetilde{\varphi}$ can be reinterpreted as criteria involving the restriction $\widehat{\varphi}$ of $\varphi$ on

$$
\mathcal{S}_{\ell_{1}, \ell_{2}}=\left\{(t, s) ; t^{2 \ell_{2}}+s^{2 \ell_{1}}=1\right\} .
$$

(v) The condition (H2) (ii) is natural and cannot be relaxed according to the necessary conditions obtained by J.L. Journé and J.M. Trépreau [JoTre] for the subellipticity of these systems. 
Organization of the paper. The proof of our main theorem will be based on an "abstract" criterion established in [De, which will be recalled in Section 2 . After the introduction of a terminology adapted to the quasihomogeneity of the problem in Section 3, we continue with the proof of the general main theorem in three steps, starting from the analysis of the quasielliptic case in Section 4, then showing how one can localize the proof in suitable quasihomogeneous sectors in Section 5 and finishing by proving the general case in Section 6 . Section $\square$ is devoted to the particular case of an analytic function $\varphi$. In the first appendix we give the computation of a basic Jacobian, whose control is important in the verification of the abstract criterion. In the second appendix, we further discuss the comparison between the criteria of hypoellipticity, subellipticity and maximal hypoellipticity on a typical homogeneous example.

\section{DERRIDJ'S SUbELLIPTICITY CRITERION}

2.1. The statement. We now recall the criterion established in De. This involves, for a given $\alpha>0$, the following geometric escape condition on $\varphi$. We do not have in this section the restriction $n=2$.

Assumption $2.1\left(H_{+}(\alpha)\right)$. There exist open sets $\omega \subset \Omega$ and $\widetilde{\omega} \subset \omega$, with full Lebesgue measure in $\omega$, and a map $\gamma$ :

$$
\widetilde{\omega} \times[0,1] \ni(\mathbf{t}, \tau) \mapsto \gamma(\mathbf{t}, \tau) \in \Omega,
$$

such that

(i) $\gamma(\mathbf{t}, 0)=\mathbf{t} ; \gamma(\mathbf{t}, 1) \notin \omega, \forall \mathbf{t} \in \widetilde{\omega}$.

(ii) $\gamma$ is of class $C^{1}$ outside a negligible set $E$, and there exist $C_{1}>0, C_{2}>0$ and $C_{3}>0$ such that

(a)

$$
\left|\partial_{\tau} \gamma(\mathbf{t}, \tau)\right| \leq C_{2}, \forall(\mathbf{t}, \tau) \in \widetilde{\omega} \times[0,1] \backslash E
$$

$$
\left|\operatorname{det}\left(D_{\mathbf{t}} \gamma\right)(\mathbf{t}, \tau)\right| \geq \frac{1}{C_{1}},
$$

where $D_{\mathbf{t}} \gamma$ denotes the Jacobian matrix of $\gamma$ considered as a map from $\widetilde{\omega}$ into $\mathbb{R}^{2}$.

(c)

$$
\varphi(\gamma(\mathbf{t}, \tau))-\varphi(\mathbf{t}) \geq \frac{1}{C_{3}} \tau^{\alpha}, \forall(\mathbf{t}, \tau) \in \widetilde{\omega} \times[0,1] .
$$

Using this assumption, the following theorem is proved in [De].

Theorem 2.2. If $\varphi$ satisfies $\left(H_{+}(\alpha)\right)$, then the associated system (1.1) $\varphi$ is microlocally $\frac{1}{\alpha}$-subelliptic in $\{\xi>0\}$.

If $-\varphi$ satisfies $\left(H_{+}(\alpha)\right)$, then the associated system (1.1) $\varphi$ is microlocally $\frac{1}{\alpha}$ subelliptic in $\{\xi<0\}$.

If $\varphi$ and $-\varphi$ satisfy $\left(H_{+}(\alpha)\right)$, then the associated system $(1.1)_{\varphi}$ is $\frac{1}{\alpha}$-subelliptic. 
2.2. The proof. For the commodity of the reader, we reproduce the proof of [De] in the case $\xi>0$.

If $u \in C_{0}^{\infty}(\omega \times \mathbb{R})$, one can, using the partial Fourier transform (with respect to $x$ ), recover $u$ from $f=L u$ by

$$
\widehat{u}(\mathbf{t}, \xi)=-\int_{0}^{1} \exp [\xi \varphi(\gamma(\mathbf{t}, \tau))] \widehat{f}(\gamma(\mathbf{t}, \tau), \xi) \cdot\left(\partial_{\tau} \gamma\right)(\mathbf{t}, \tau) d \tau .
$$

Taking Cauchy-Schwarz in (2.2), we obtain

$$
\begin{aligned}
&|\widehat{u}(\mathbf{t}, \xi)|^{2} \\
& \leq\left(\int_{0}^{1} \exp [\xi \varphi(\gamma(\mathbf{t}, \tau))] d \tau\right) \\
& \quad \times\left(\int_{0}^{1} \exp [\xi \varphi(\gamma(\mathbf{t}, \tau))]\left|\widehat{f}(\gamma(\mathbf{t}, \tau), \xi) \cdot\left(\partial_{\tau} \gamma\right)(\mathbf{t}, \tau)\right|^{2} d \tau\right) .
\end{aligned}
$$

By items (ii) (a) and (ii) $)_{(\mathrm{c})}$ in Assumption 2.1, this implies

$$
|\widehat{u}(\mathbf{t}, \xi)|^{2} \leq C_{2}^{2} \quad\left(\int_{0}^{1} \exp -\frac{1}{C_{3}} \tau^{\alpha} \xi d \tau\right) \times\left(\int_{0}^{1} \exp -\frac{1}{C_{3}} \tau^{\alpha} \xi|\widehat{f}(\gamma(\mathbf{t}, \tau), \xi)|^{2} d \tau\right) .
$$

So, integrating in $\mathbf{t}$ over $\omega$, we get

$$
\begin{aligned}
& \int_{\omega}|\widehat{u}(\mathbf{t}, \xi)|^{2} d \mathbf{t} \\
& \quad \leq C_{2}^{2} \quad\left(\int_{0}^{1} \exp -\frac{1}{C_{3}} \tau^{\alpha} \xi d \tau\right) \times\left(\int_{0}^{1} \int_{\omega} \exp -\frac{1}{C_{3}} \tau^{\alpha} \xi|\widehat{f}(\gamma(\mathbf{t}, \tau), \xi)|^{2} d \mathbf{t} d \tau\right) .
\end{aligned}
$$

We now change variables: $\mathbf{v}=\gamma(\mathbf{t}, \tau), \tau=\tau$. The second term in the r.h.s. of (2.4) can be estimated as follows:

$$
\begin{aligned}
& \int_{0}^{1} \int_{\omega}\left(\exp -\frac{1}{C_{3}} \tau^{\alpha} \xi\right)|\widehat{f}(\gamma(\mathbf{t}, \tau), \xi)|^{2} d \mathbf{t} d \tau \\
& \quad=\int_{0}^{1} \int_{\omega}\left(\exp -\frac{1}{C_{3}} \tau^{\alpha} \xi\right)|\widehat{f}(\mathbf{v}, \xi)|^{2}\left|D_{\mathbf{t}} \gamma\right|^{-1} d \tau d \mathbf{v} \\
& \quad \leq C_{1}\left(\int_{0}^{1} \exp -\frac{1}{C_{3}} \tau^{\alpha} \xi d \tau\right)\left(\int_{\Omega}|\widehat{f}(\mathbf{v}, \xi)|^{2} d \mathbf{v}\right)
\end{aligned}
$$

where we have used the lower bound (2.1) for the Jacobian $\left|D_{\mathbf{t}} \gamma\right|$.

So finally, one has

$$
\int_{\omega}|\widehat{u}(\mathbf{t}, \xi)|^{2} d \mathbf{t} \leq C_{1} C_{2}^{2}\left(\int_{0}^{1}\left(\exp -\frac{1}{C_{3}} \tau^{\alpha} \xi\right) d \tau\right)^{2}\left(\int_{\Omega}|\widehat{f}(\mathbf{v}, \xi)|^{2} d \mathbf{v}\right) .
$$

We then obtain the existence of $C\left(C_{1}, C_{2}, C_{3}\right)>0$ such that, for all $\xi>0$,

$$
\int_{\omega}|\widehat{u}(\mathbf{t}, \xi)|^{2} d \mathbf{t} \leq C\left(C_{1}, C_{2}, C_{3}\right)|\xi|^{-\frac{2}{\alpha}}\left(\int_{\Omega}|\widehat{f}(\mathbf{v}, \xi)|^{2} d \mathbf{v}\right) .
$$

\section{Quasihomogeneous Structure}

3.1. Distorted geometry. Condition (i) in Assumption 2.1 expresses the property that the curve is escaping from $\omega$. For the description of escaping curves, it appears useful to extend the usual terminology used in the Euclidean space $\mathbb{R}^{2}$ in a way which is adapted to the given quasihomogeneous structure. This is realized by introducing the dressing map:

$$
(t, s) \mapsto d_{\ell}(t, s)=\left(t|t|^{\ell-1}, s\right),
$$

which is at least of class $C^{1}$ as $\ell \geq 1$, and whose main role is to transport the distorted geometry onto the Euclidean geometry. 
The first example was the unit distorted circle (in short unit disto-circle or unit "circle") $\mathcal{S}$ introduced in (1.9) whose image by $d_{\ell}$ becomes the standard unit circle in $\mathbb{R}^{2}$ centered at $(0,0)$.

Similarly, we will speak of disto-sectors, disto-arcs, disto-rays, disto-disks. In particular, for $(a, b) \in \mathcal{S}$, we define the disto-ray $\mathcal{R}_{(a, b)}$ by

$$
\mathcal{R}_{(a, b)}:=\left\{\left(\lambda a, \lambda^{\ell} b\right) ; 0 \leq \lambda \leq 1\right\} .
$$

The disto-scalar product of two vectors in $\mathbb{R}^{2},(t, s)$ and $\left(t^{\prime}, s^{\prime}\right)$, is then given by

$$
\left\langle(t, s) \mid\left(t^{\prime}, s^{\prime}\right)\right\rangle_{\ell}=t t^{\prime}\left|t t^{\prime}\right|^{\ell-1}+s s^{\prime}
$$

(for $\ell=1$, we recover the standard scalar product).

For $(t, s) \in \mathbb{R}^{2}$, we also introduce the quasihomogeneous positive function $\varrho$ defined on $\mathbb{R}^{2}$ by

$$
\varrho(t, s)^{2 \ell}=t^{2 \ell}+s^{2} .
$$

With this notation, we observe that, if $(t, s) \in \mathbb{R}^{2} \backslash\{(0,0)\}$, then

$$
(\widetilde{t}, \widetilde{s}):=\left(\frac{t}{\varrho(t, s)}, \frac{s}{\varrho(t, s)}\right) \in \mathcal{S}
$$

and

$$
(t, s) \in \mathcal{R}_{(\tilde{t}, \widetilde{s})} .
$$

The open disto-disk $D(R)$ is then defined by

$$
D(R)=\{(x, y) \mid \varrho(x, y)<R\} .
$$

We can also consider a parametrization of the disto-circle by a parameter on the corresponding circle $\vartheta \in \mathbb{R} / 2 \pi \mathbb{Z}$ (through the dressing map). We note that we have a natural (anticlockwise) orientation of the disto-circle. In other cases it will be better to parametrize by $s$ (if $t \neq 0$ ) or by $t$ (if $s \neq 0$ ). So a point in $\mathcal{S}$ will be defined either by $\theta$, by $(a, b) \in \mathbb{R}^{2}$ or by $\vartheta$.

Once an orientation is defined on $\mathcal{S}$, two points $\theta_{1}$ and $\theta_{2}$ (or $\left(a_{1}, b_{1}\right)$ and $\left.\left(a_{2}, b_{2}\right)\right)$ on $\mathcal{S}$ will determine a unique unit "sector" $V \subset D(1)$.

3.2. Distorted dynamics. The parametrized curves $\gamma$ permitting us to satisfy Assumption 2.1 will actually be "lines" (possibly broken) which will finally escape from a neighborhood of the origin. Our aim in this subsection is to define these "lines" (actually distorted parametrized lines).

In parametric coordinates, with

$$
t(\tau)=t+\nu \tau,
$$

the curve $\gamma$ starting from $(t, s)$ and disto-parallel to $(c, d)$ is defined by writing that the vector $\left(t(\tau)|t(\tau)|^{\ell-1}-t|t|^{\ell-1}, s(\tau)-s\right)$ is parallel to $\left(c|c|^{\ell-1}, d\right)$.

In the applications, we will only consider $\nu= \pm c$. So

$$
\left(t(\tau)|t(\tau)|^{\ell-1}-t|t|^{\ell-1}\right) d=c|c|^{\ell-1}(s(\tau)-s),
$$

and we find

$$
s(\tau)=s+\frac{d}{c|c|^{\ell-1}}\left(t(\tau)|t(\tau)|^{\ell-1}-t|t|^{\ell-1}\right) .
$$

We consider the map $\sigma \mapsto f_{\ell}(\sigma)$ which is defined by

$$
f_{\ell}(\sigma)=\sigma|\sigma|^{\ell-1} \text {. }
$$


Note that

$$
f_{\ell}^{\prime}(\sigma)=\ell|\sigma|^{\ell-1} \geq 0 .
$$

With this new function, (3.8) can be written as

$$
d f_{\ell}(t(\tau))-s(\tau) f_{\ell}(c)=d f_{\ell}(t)-s f_{\ell}(c) .
$$

This leads us to use the notion of a distorted determinant of two vectors in $\mathbb{R}^{2}$. For two vectors $v:=\left(v_{1}, v_{2}\right)$ and $w:=\left(w_{1}, w_{2}\right)$, we introduce

$$
\Delta_{\ell}(v ; w)=f_{\ell}\left(v_{1}\right) w_{2}-v_{2} f_{\ell}\left(w_{1}\right) .
$$

We will also write

$$
\Delta_{\ell}(v ; w)=\Delta_{\ell}\left(v_{1}, v_{2}, w_{1}, w_{2}\right) .
$$

With this notation, (3.11) can be written as

$$
\Delta_{\ell}((c, d) ;(t(\tau), s(\tau)))=\Delta_{\ell}((c, d) ;(t, s))
$$

or

$$
\Delta_{\ell}(c, d, t(\tau), s(\tau))=\Delta_{\ell}(c, d, t, s) .
$$

When $\ell=1$, we recover the usual determinant of two vectors in $\mathbb{R}^{2}$. For $\ell \geq 1$, we simply have the relation

$$
\Delta_{\ell}(v ; w)=\Delta_{1}\left(d_{\ell}(v) ; d_{\ell}(w)\right) .
$$

Note that $\Delta_{\ell}(v ; w)$ vanishes when $d_{\ell}(v)$ and $d_{\ell}(w)$ are collinear.

We now look at the variation of $\psi$ which is defined (for a given initial point $(t, s)$ ) by

$$
\tau \mapsto \psi(\tau)=\rho(\tau)^{2 \ell}=t(\tau)^{2 \ell}+s(\tau)^{2},
$$

with

$$
\rho(\tau):=\varrho(t(\tau), s(\tau)) .
$$

Easy computations also give

$$
\psi^{\prime}(\tau)=\frac{2 \nu}{f_{\ell}(c)} f_{\ell}^{\prime}(t+\nu \tau)\langle(c, d) \mid(t(\tau), s(\tau))\rangle_{\ell},
$$

whose sign is the product of the sign of the (disto)-scalar product of $(c, d)$ and $(t(\tau), s(\tau))$ and the sign of $c \nu$.

We now analyze the variation of the (disto)-scalar product $\langle(c, d) \mid(t(\tau), s(\tau))\rangle_{\ell}$ as a function of $\tau$. We have the formula

$$
\langle(c, d) \mid(t(\tau), s(\tau))\rangle_{\ell}=\langle(c, d) \mid(t, s)\rangle_{\ell}+\frac{1}{f_{\ell}(c)}\left(f_{\ell}(t(\tau))-f_{\ell}(t)\right) .
$$

If we now assume that

$$
c \nu>0,\langle(c, d) \mid(a, b)\rangle_{\ell} \geq 0,
$$

then for $(s, t)$ in the unit sector $\mathcal{V}_{a b c d}$ associated to the arc $((a, b),(c, d))$, we obtain

$$
\psi^{\prime}(\tau) \geq \frac{1}{f_{\ell}(c)^{2}} \times\left(2 \nu f_{\ell}^{\prime}(t+\nu \tau)\right) \times\left(f_{\ell}(t(\tau))-f_{\ell}(t)\right) .
$$

We rewrite this inequality in the form

$$
\psi^{\prime}(\sigma) \geq \frac{1}{f_{\ell}(c)^{2}} \times\left(\left(f_{\ell}(t(\sigma))-f_{\ell}(t)\right)^{2}\right)^{\prime}, \forall \sigma \geq 0 .
$$


Integrating over $[0, \tau]$, we get for $\tau \geq 0$ :

$$
\psi(\tau) \geq \frac{1}{f_{\ell}(c)^{2}} \times\left(f_{\ell}(t(\tau))-f_{\ell}(t)\right)^{2} .
$$

We now need the following

Lemma 3.1. For any $\ell \geq 1, \tau \geq 0$, and $\gamma \in \mathbb{R}$, we have

$$
f_{\ell}(\tau+\gamma)-f_{\ell}(\gamma) \geq f_{\ell}\left(\frac{\tau}{2}\right) .
$$

Remark 3.2. This lemma can be improved when $\gamma \geq 0$; we can then show

$$
f_{\ell}(\tau+\gamma)-f_{\ell}(\gamma) \geq f_{\ell}(\tau) .
$$

Proof. By the previous remark, the proof is clear when $\gamma \geq 0$ or when $\gamma+\tau \leq 0$. So it remains to analyze the case when $-\tau<\gamma<0$. But the two terms on the left hand side are now positive. So we immediately obtain (3.23) by observing that $\max (\tau+\gamma,-\gamma) \geq \frac{\tau}{2}$.

Remark 3.3. If we take the square, we obtain (and this time for any $\tau \in \mathbb{R}$ ) the inequality

$$
\left(f_{\ell}(\tau+\gamma)-f_{\ell}(\gamma)\right)^{2} \geq\left(\frac{\tau}{2}\right)^{2 \ell} .
$$

Now using Lemma 3.1 this leads to

Lemma 3.4. Under condition (3.21), we have, for any $\tau \geq 0$ and for any $(t, s) \in$ $\mathcal{V}_{a b c d}$,

$$
\rho(\tau)^{2 \ell}-\rho(0)^{2 \ell} \geq\left(\frac{\nu \tau}{2 c}\right)^{2 \ell} .
$$

If instead $\nu c<0$, then we obtain

$$
\rho(\tau)^{2 \ell}-\rho(0)^{2 \ell} \leq-\left(\frac{\nu \tau}{2 c}\right)^{2 \ell} .
$$

We continue by analyzing the variation of $s(\tau)$ and $t(\tau)$ and more precisely the variation on the disto-circle of

$$
\widetilde{t}(\tau)=\frac{t(\tau)}{\rho(\tau)}, \widetilde{s}(\tau)=\frac{s(\tau)}{\rho(\tau)^{\ell}} .
$$

After some computations, we get, with

$$
\begin{gathered}
\nu= \pm c, \\
\widetilde{t}^{\prime}(\tau)= \pm|c|^{1-\ell} \frac{s(\tau)}{\rho(\tau)^{2 \ell+1}} \Delta_{\ell}(c, d, t, s),
\end{gathered}
$$

which can also be written in the form

$$
\widetilde{t}^{\prime}(\tau)= \pm|c|^{1-\ell} \frac{\widetilde{s}(\tau)}{\rho(\tau)} \Delta_{\ell}(c, d, \widetilde{t}(\tau), \widetilde{s}(\tau)) .
$$

Similarly, we get for $\widetilde{s}^{\prime}$,

$$
\widetilde{s}^{\prime}(\tau)=\mp \ell|c|^{1-\ell} \frac{t(\tau)^{2 \ell-1}}{\rho(\tau)^{3 \ell}} \Delta_{\ell}(c, d, t, s)
$$

and

$$
\widetilde{s}^{\prime}(\tau)=\mp \ell|c|^{1-\ell} \frac{\widetilde{t}^{2 \ell-1}(\tau)}{\rho(\tau)} \Delta_{\ell}(c, d, \widetilde{t}(\tau), \widetilde{s}(\tau)) .
$$




\section{Analysis of the QUasielliptic Case $(\widetilde{\varphi}>0)$}

We first start the proof of the main theorem with the particular case when

$$
\widetilde{\varphi} \geq \mu>0 \text {. }
$$

This case is already interesting for presenting the main ingredients of the general proof. We can remark indeed that what we are doing below in $\mathcal{S}$ can be done later in a specific (disto)-arc of $\mathcal{S}$.

In $\mathcal{S}$, taking a regular parametrization of $\mathcal{S}$ denoted by $\theta$, we consider the connected components of $\left\{\widetilde{\varphi}^{\prime} \geq 0\right\}$ or of $\left\{\widetilde{\varphi}^{\prime} \leq 0\right\}$.

Our assumption takes in this case the following form.

Assumption 4.1 $\left(H_{1}\right)$. Either the cardinal of the connected components in $\mathcal{S}$ of $\left\{\widetilde{\varphi}^{\prime} \geq 0\right\}$ or the cardinal of the connected components of $\left\{\widetilde{\varphi}^{\prime} \leq 0\right\}$ is finite.

Remark 4.2. This assumption is automatically satisfied if $\widetilde{\varphi}$ is analytic.

Theorem 4.3. Let $\varphi$ in $C^{1}\left(\mathbb{R}^{2} ; \mathbb{R}\right)$ satisfy (1.7) and (4.1). Then $\left(H_{1}\right)$ implies $\left(H_{+}(\alpha)\right)$, with $\alpha=\frac{1}{m}$.

Corollary 4.4. If $\left(H_{1}\right)$ is satisfied, then the system (1.1) is $\frac{1}{m}$-subelliptic in $\{\xi>0\}$.

\section{Proof of Theorem 4.3 .}

Step 1. Construction of the covering.

Under Assumption (H1) (say for definiteness under the first alternative), and considering the connected components introduced there, we first start by constructing a finite covering of $\mathcal{S}$ by a family of disjoints open $\operatorname{arcs} \mathcal{S}_{j}$ such that

$$
\mathcal{S}=\bigcup_{j} \overline{\mathcal{S}_{j}}
$$

in the following way. If $\theta_{j}$ (or $\left.\left(a_{j}, b_{j}\right)\right)$ denotes the sequence of the left end points of the components of $\left\{\widetilde{\varphi}^{\prime} \geq 0\right\}$ and by $\widehat{\theta}_{j}$ (or $\left.\left(c_{j}, d_{j}\right)\right)$ the sequence of the right end points, we define $\mathcal{S}_{j}$ as the arc $\mathcal{S}_{j}=\left(\theta_{j}, \theta_{j+1}\right)$. We observe that:

$\widetilde{\varphi}$ is nondecreasing on $\left(\theta_{j}, \widehat{\theta}_{j}\right)$ and strictly decreasing on $\left(\widehat{\theta}_{j}, \theta_{j+1}\right)$.

We now associate to the (disto)-arc $\mathcal{S}_{j}$ its (disto)-unit sector $\mathcal{V}_{j}$. For technical reasons, we will add a finite number of points such that we finally have a (possibly new) family of open $\operatorname{arcs} \mathcal{S}_{j}=\left(\theta_{j}, \theta_{j+1}\right)$ such that each arc satisfies Property 1.2.

Step 2. Construction of $\gamma$.

We construct $\gamma$ independently in each sector $\mathcal{V}_{j}$. More precisely, this will be a map from $\left[\left(\mathcal{V}_{j} \cup \mathcal{R}_{\theta_{j}}\right) \backslash\{(0,0)\}\right] \times[0,1]$ into $\Omega$ (and actually in the infinite sector associated to $\mathcal{S}_{j}$ ). From now on in this paragraph, we fix $j$ (and make it equal to $1)$. So $\mathcal{S}_{1}$ denotes the set of points $(t, s) \in D(1)$ such that $\left(\frac{t}{\rho(t, s)}, \frac{s}{\rho(t, s)^{\ell}}\right) \in\left(\theta_{1}, \theta_{2}\right)$.

We now define $\gamma$ (see (3.7)-(3.8), with $\nu=c_{1}$ ) by

(i) If $c_{1} \neq 0$,

$$
\gamma(t, s, \tau):=(t(\tau), s(\tau)):=\left(t+c_{1} \tau, s+\frac{d_{1}}{f_{\ell}\left(c_{1}\right)}\left(f_{\ell}(t(\tau))-f_{\ell}(t)\right) .\right.
$$

(ii) If $c_{1}=0$

$$
\gamma(t, s, \tau):=(t(\tau), s(\tau)):=\left(t, s+d_{1} \tau\right) .
$$


Remarks 4.5. (i) Note that for any $\left(t_{0}, s_{0}, \tau\right)$ the Jacobian of the map $(t, s) \mapsto$ $\gamma(t, s, \tau)$ at $\left(t_{0}, s_{0}\right)$ is 1.

(ii) Actually, one can avoid the second case by replacing $c_{1}=0$ by an arbitrarily close $\widetilde{c}_{1}$ whose sign will depend on the considered "sector".

From now on, we write for simplification $c=c_{1}, d=d_{1}$.

Let us look at the most generic case when $c \neq 0$. In order to show $\left(H_{+}(\alpha)\right)$, the only nontrivial property is to show property (ii) $)_{(\mathrm{c})}$ in Assumption 2.1.

Lemma 4.6. If $(t, s)$ belongs to the "subsector" associated with $\left[\theta_{1}, \widehat{\theta}_{1}\right]$ (resp. to the "subsector" associated with $\left.\left[\widehat{\theta}_{1}, \theta_{2}\right]\right)$, then the whole curve $\gamma(t, s, \tau)$ stays in the same (infinite) "subsector" for $\tau \geq 0$.

Proof. The lemma is geometrically evident using the dressing map $d_{\ell}$.

Step 3. Lower bound along the curve $\gamma$.

Let us add the notation

$$
\rho(\tau)=\varrho(\gamma(t, s, \tau)), \rho=\rho(0)
$$

and

$$
\theta(\tau)=\theta_{\tau}=\left(\frac{t(\tau)}{\rho(\tau)}, \frac{s(\tau)}{\rho^{\ell}(\tau)}\right)=(\widetilde{t}(\tau), \widetilde{s}(\tau)),
$$

with $\theta=\theta(0)=(\widetilde{t}, \widetilde{s})$.

We note that with the above notation

$$
\varphi(t, s)=\rho^{m} \widetilde{\varphi}(\widetilde{t}, \widetilde{s}) .
$$

We want to show (ii) (c) in Assumption 2.1 and first decompose the expression which has to be estimated from below by writing

$$
\rho(\tau)^{m} \widetilde{\varphi}\left(\theta_{\tau}\right)-\rho^{m} \widetilde{\varphi}(\theta)=\left(\rho(\tau)^{m}-\rho^{m}\right) \widetilde{\varphi}(\theta)+\rho(\tau)^{m}\left(\widetilde{\varphi}\left(\theta_{\tau}\right)-\widetilde{\varphi}(\theta)\right),
$$

and will then obtain a lower bound for each term of the r.h.s. of (4.8).

Analysis of $\rho(\tau)^{m}-\rho^{m}$. Using (3.26) and

$$
\rho(\tau)^{m}-\rho^{m}=\left(\rho(\tau)^{2 \ell}\right)^{\frac{m}{2 \ell}}-\left(\rho^{2 \ell}\right)^{\frac{m}{2 \ell}} \geq\left(\rho(\tau)^{2 \ell}-\rho^{2 \ell}\right)^{\frac{m}{2 \ell}},
$$

where we note that $m \geq 2 \ell$, we deduce

$$
\rho(\tau)^{m}-\rho^{m} \geq 2^{-m} \tau^{m}, \forall \tau \geq 0 .
$$

Finally 3 using the lower bound for $\widetilde{\varphi}$, we get for the first term of the r.h.s. of (4.8) the inequality

$$
\left(\rho(\tau)^{m}-\rho^{m}\right) \widetilde{\varphi}(\theta) \geq 2^{-m} \mu \tau^{m} .
$$

Analysis of $\widetilde{\varphi}\left(\theta_{\tau}\right)-\widetilde{\varphi}(\theta)$. Having in mind our assumptions on the variation of $\widetilde{\varphi}$ on $\left[\theta_{1}, \theta_{2}\right]$, we have simply to prove here:

- If $(\widetilde{t}, \widetilde{s}) \in\left[\theta_{1}, \widehat{\theta}_{1}\right]$, the function $\tau \mapsto \theta_{\tau}$ is nondecreasing.

- If $(\widetilde{t}, \widetilde{s}) \in\left[\widehat{\theta}_{1}, \theta_{2}\right]$, the function $\tau \mapsto \theta_{\tau}$ is nonincreasing.

But this is immediate after having sent the initial picture by the dressing map (3.1).

\footnotetext{
${ }^{2}$ We will add in footnotes some indications for the other case.

${ }^{3}$ In the case when $c=0$, if $d s>0$, we have

$$
\psi(\tau)=(s+d \tau)^{2}-s^{2} \geq \tau^{2} \geq \tau^{2 \ell}, \forall \tau \in[0,1] .
$$
}


5. Control in the CASE of the SeCtors $\mathcal{V}_{j}^{+} \cup \mathcal{R}_{\left(a_{j}, b_{j}\right)}$

Provisory assumption. For the control of the order of the zeroes, we assume for the moment that the $p$ appearing in (1.10) (Assumption 1.1) satisfies

$$
1 \leq p \leq m \text {. }
$$

This will be removed later (see Subsection 6.3).

In comparison with the quasielliptic situation, the only point is that the condition of positivity could be not satisfied at one or two ends. Note that we keep Assumption 1.1 (iii). The essential idea is then to improve the second part of the lower bound for (4.8) using the fifth item of Assumption 1.1 (i.e. the lower bound $(1.10)$ ).

Keeping in mind what we have done before, it remains to analyze "rays" coming from points $(t, s)$ which are close to $\mathcal{R}_{\left(a_{j}, b_{j}\right)}$. The key estimate is that there exists $\mu_{0}>0$ and a "sector" $\mathcal{V}$ neighboring this "end-ray" such that

$$
\widetilde{\varphi}\left(\theta_{\tau}\right)-\widetilde{\varphi}(\theta) \geq \mu_{0}\left(\frac{\tau}{\rho(\tau)}\right)^{m}, \forall(t, s) \in \mathcal{V}, \forall \tau \in[0,1] .
$$

But this proof is immediate from (1.10) if we control more precisely the positivity of $\theta(\tau)-\theta(0)$, as we shall see in (5.9).

Quantitative control of the positivity. We treat three typical cases.

Case 1. $c>0, d>0, a_{1}>0, b_{1}<0$.

We assume that $\widetilde{\varphi}\left(a_{1}, b_{1}\right)=0$, and it is enough to control the trajectories starting from $(t, s) \in \sigma$ where the "sector" $\sigma$ is defined by the condition that the corresponding $(\widetilde{t}, \widetilde{s})$ is close to $\left(a_{1}, b_{1}\right)$ and between $\left(a_{1}, b_{1}\right)$ and $(c, d)$ :

$$
(t, s) \in \sigma \text { iff }(\widetilde{t}, \widetilde{s}) \in\left(\theta_{1}, \theta_{1}+\epsilon_{1}\right)
$$

for some $\epsilon_{1}>0$.

The point here is that one has a regular parametrization of the disto-circle by the $\tilde{s}$ variable and that we stay in the half-right plan.

By formula (3.31), we observe that for $(t, s)$ as before, we have

$$
\widetilde{s}^{\prime}(\tau) \geq 0, \forall \tau \in[0,1] .
$$

Here we have used the fact that $\Delta_{\ell}\left(c, d, a_{1}, b_{1}\right)<0$. We also get

$$
\widetilde{\varphi}\left(\theta_{\tau}\right)-\widetilde{\varphi}(\theta) \geq 0 \text {. }
$$

We now use (3.32) and observe that $\Delta_{\ell}(\widetilde{t}(\tau), \widetilde{s}(\tau), c, d)$ is increasing as $\tau$ increases (together with $\widetilde{s}(\tau)$ ).

Let us observe the trivial inequality

$$
1 \geq \frac{t(\tau)}{\rho(\tau)} \geq c \frac{\tau}{\rho(\tau)} .
$$

So there exists $\rho$ and for any $(s, t) \in \sigma$, there exists $\tau(t, s)$ such that

$$
\Delta_{\ell}((\widetilde{t}(\tau), \widetilde{s}(\tau)) ;(c, d)) \geq \rho,
$$

for all $\tau \in[0, \tau(t, s)]$ and $\Delta_{\ell}((\widetilde{t}(\tau), \widetilde{s}(\tau)) ;(c, d)) \leq \rho$ if $\tau \in[\tau(t, s), 1]$. 
In the first interval, we observe that $\widetilde{t}$ is semibounded on $\sigma$, so

$$
\widetilde{s}(\tau)-\widetilde{s}(0) \geq \mu_{1} \frac{\tau}{\rho(\tau)}, \forall \tau \in[0, \tau(t, s)],
$$

where $\mu_{1}=\ell\left(\inf _{(t, s) \in \sigma} \widetilde{t}^{2 \ell-1}\right) c^{1-\ell} \rho$.

This gives a uniform lower bound for $\tau \in[0, \tau(t, s)]$, but for $\tau \geq \tau(t, s)$ we have a uniform lower bound (if we choose $\rho$ small enough) of $\widetilde{s}(\tau)-\widetilde{s}(0)$ by a constant $\alpha_{\rho}>0$. This together with (5.5) gives the existence of $\mu_{2}>0$ such that

$$
\widetilde{s}(\tau)-\widetilde{s}(0) \geq \mu_{2} \frac{\tau}{\rho(\tau)}, \forall \tau \in[\tau(t, s), 1] .
$$

So we have finally shown that there exists $\mu_{\sigma}>0$ such that

$$
\widetilde{s}(\tau)-\widetilde{s}(0) \geq \mu_{\sigma} \frac{\tau}{\rho(\tau)}, \forall \tau \in[0,1] .
$$

Case 2. $c>0, d>0, a_{2}<0, b_{2}>0$.

We assume that $\widetilde{\varphi}\left(a_{2}, b_{2}\right)=0$ and observe that it is enough to control the trajectories starting from $(s, t)$ with corresponding $(\widetilde{s}(0), \widetilde{t}(0))$ close to $\left(a_{2}, b_{2}\right)$ and between $(c, d)$ and $\left(a_{2}, b_{2}\right)$.

Here $\widetilde{t}(\tau)$ may change sign along the trajectory, and we must parametrize the disto-circle by the variable $\widetilde{t}$.

Here we use (3.29) for observing that $\widetilde{t}(\tau)$ is this time increasing (note that $\Delta_{\ell}\left(c, d, a_{2}, b_{2}\right)>0$ and $\left.s(\tau) \geq \varrho_{0}>0\right)$.

A similar argument to the one leading to (5.6) gives the uniform control of $|\widetilde{t}(\tau)-\widetilde{t}(0)|$ from below. Here we can no longer use (5.5) but will instead use (3.26) which implies

$$
\frac{\tau}{\rho(\tau)} \leq 2
$$

without assuming the positivity of $t$.

There are limiting cases when we shall use both parametrizations, but this does not lead to any new difficulties. Typically, let us consider the following case.

Case 3. $c=0, d=-1, a_{1}<0, b_{1}<0$.

We just have to use in this case vertical escaping "rays".

So in all the cases we obtain that, if we start (inside $\mathcal{V}_{j}^{+}$) with a "subsector" $\sigma$, whose closure does not meet the "ray" $\mathcal{R}_{(c, d)}$, then there exists $\mu_{\sigma}>0$ such that

$$
|\theta(\tau)-\theta(0)| \geq \mu_{\sigma} \frac{\tau}{\rho(\tau)}, \forall \tau \in[0,1] .
$$

So we have finally proven

Lemma 5.1. Assuming that $\widetilde{\varphi}$ satisfies in $\mathcal{V}_{j}^{+}$Property 1.2 and the nondegeneracy Assumption (1.10) for its zero possibly appearing at $\left(a_{j}, b_{j}\right)$, then there exists $\mu>0$ such that

$$
\rho(\tau)^{m} \varphi(\theta(\tau))-\rho(0)^{m} \varphi(\theta(0)) \geq \mu \tau^{m}, \forall \tau \in[0,1],
$$

with $(\rho(0), \theta(0))$ corresponding to a point of $\mathcal{V}_{j}^{+} \cup \mathcal{R}_{\left(a_{j}, b_{j}\right)}$.

Remark 5.2. A similar result can be obtained for $\mathcal{V}_{j}^{+} \cup \mathcal{R}_{\left(a_{j+1}, b_{j+1}\right)}$ with a zero at $\left(a_{j+1}, b_{j+1}\right)$. 


\section{Control For the "SECtors" Where $\widetilde{\varphi}$ IS NONPOSitive}

6.1. Main case. We consider first the case of small opening "sectors" $\mathcal{V}_{k}^{-}$. More precisely, we assume that

$$
\left\langle(c, d) \mid\left(a_{j}, b_{j}\right)\right\rangle_{\ell}>0, \text { for } j=k, k+1 .
$$

6.1.1. The subcase $c \neq 0$. We start with the case when

$$
c \neq 0 \text {. }
$$

We keep the same notation (change the labelling by taking $k=1$ ), but this time $(c, d)$ is a point of $\mathcal{S}$ where $\widetilde{\varphi}$ is a minimum. The map $\gamma_{-}$is defined as follows.

If $(t, s) \in D\left(\frac{1}{4}\right)$ is such that $(\widetilde{t}, \widetilde{s}) \in\left(\left(a_{1}, b_{1}\right),(c, d)\right)$, we let

(i) $\gamma_{-}=\gamma_{1}$ with

$$
\begin{aligned}
\gamma_{1}(t, s, \tau) & :=(t(\tau), s(\tau)) \\
& :=\left(t-c \tau, s+\frac{d}{f_{\ell}(c)}\left(f_{\ell}(t(\tau))-f_{\ell}(t)\right)\right), \forall \tau \in\left[0, \tau_{1}(t, s)\right],
\end{aligned}
$$

where $\tau_{1}(t, s)=\tau_{1}$ is the smallest $\tau$ such that $\gamma_{1}(t, s, \tau) \in \mathcal{R}_{\left(a_{1}, b_{1}\right)}$.

(ii) $\gamma_{-}=\gamma_{2}$ with

$$
\gamma_{2}(t, s, \tau)=\gamma_{+}\left(t\left(\tau_{1}\right), s\left(\tau_{1}\right), \tau-\tau_{1}\right), \forall \tau \in\left[\tau_{1}, 1\right],
$$

where $\gamma_{+}$is the map constructed in (4.3) in the "sector" $\mathcal{V}_{0}^{+}$(the "sector" preceding $\mathcal{V}_{1}$ when turning anticlockwise).

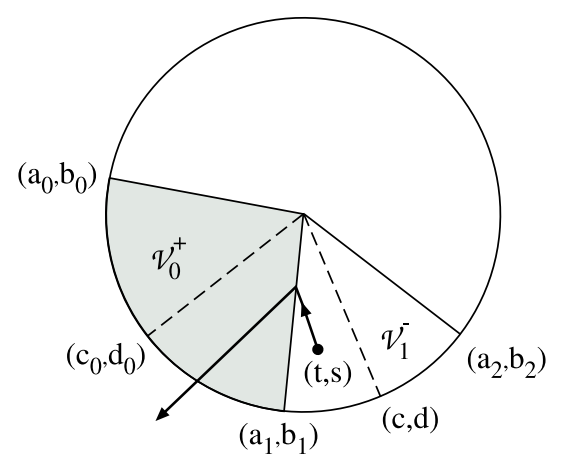

FiguRE 1. The escaping ray (after application of the dressing map)

If $(\widetilde{t}, \widetilde{s}) \in\left((c, d),\left(a_{2}, b_{2}\right)\right)$, we do the same construction with $\mathcal{R}_{\left(a_{1}, b_{1}\right)}$ replaced by $\mathcal{R}_{\left(a_{2}, b_{2}\right)}$.

Let us compute $\tau_{1}(t, s)$. This $\tau_{1}$ is actually determined by writing that, at the corresponding point $\left(t\left(\tau_{1}\right), s\left(\tau_{1}\right)\right)=\left(t_{1}(t, s), s_{1}(t, s)\right)$, we should have

$$
\Delta_{\ell}\left(a_{1}, b_{1}, t_{1}(t, s), s_{1}(t, s)\right)=0
$$

and

$$
\Delta_{\ell}\left(c, d, t_{1}(t, s), s_{1}(t, s)\right)=\Delta_{\ell}(c, d, t, s) .
$$

The first one (6.5) expresses that we cross $\mathcal{R}_{a_{1}, b_{1}}$, and the second one (6.6) was observed in (3.11). This leads to the determination of $\tau_{1}$ by the formula

$$
f_{\ell}\left(t-c \tau_{1}\right)=f_{\ell}\left(a_{1}\right) \frac{\Delta_{\ell}(t, s, c, d)}{\Delta_{\ell}\left(a_{1}, b_{1}, c, d\right)} .
$$


The uniqueness is obtained by the monotonicity of $\tau \mapsto f_{\ell}\left(t-c \tau_{1}\right)$ for $c \neq 0$, and the existence is a consequence of the transversality of $\mathcal{R}_{a_{1}, b_{1}}$ and the disto-parallel to $\mathcal{R}_{c, d}$ which is expressed by the condition

$$
\Delta_{\ell}\left(a_{1}, b_{1}, c, d\right) \neq 0 .
$$

It remains to control the Jacobian of the map $\gamma$. It is immediate to see that the Jacobian of $(t, s) \mapsto \gamma(t, s, \tau)$ is equal to one when $\tau<\tau_{1}(t, s)$. Let us look at the more difficult case when

$$
\tau>\tau_{1}(t, s)
$$

Under this condition, we write

$$
\gamma_{2}(t, s, \tau)=\left(t_{2}(t, s, \tau), s_{2}(t, s, \tau)\right)
$$

but omit the reference to $\tau$ in the computations below and prefer to think of $t_{1}, t_{2}$ and $\tau_{1}$ as functions of $(t, s)$.

In order to compute the Jacobian, it is enough to compare the two 2-forms $d t_{2} \wedge d s_{2}$ and $d t \wedge d s$. We have in addition to (6.5) and (6.6)

$$
\begin{aligned}
& t_{1}(t, s)=t-c \tau_{1}(t, s) \\
& t_{2}(t, s)=t_{1}(t, s)+\hat{c}\left(\tau-\tau_{1}(t, s)\right), \\
& s_{2}(t, s)=s_{1}(t, s)+\frac{\hat{d}}{f_{\ell}(\hat{c})}\left(f_{\ell}\left(t_{2}(t, s)\right)-f_{\ell}\left(t_{1}(t, s)\right)\right) .
\end{aligned}
$$

Here $(\hat{c}, \hat{d})$ denotes the coordinates (and we assume for the moment 4 that $\hat{c} \neq 0$ ) of the maximum of $\widetilde{\varphi}$ in the arc (corresponding to $\mathcal{V}_{k-1}^{+}$in the initial notation) preceding the arc $\left(\left(a_{1}, b_{1}\right),\left(a_{2}, b_{2}\right)\right)$.

The two first identities imply first (by (6.5) that

$$
d t_{1} \wedge d s_{1}=0
$$

and that (by (6.5) and (6.6) ) one can express the 1 -forms $d s_{1}$ and $t_{1}(t, s)^{\ell-1} d t_{1}$ as linear combinations of the 1 -forms $d s$ and $f_{\ell}^{\prime}(t) d t$. In particular we get

$$
d s_{1}=\frac{b_{1}}{f_{\ell}\left(a_{1}\right)} f_{\ell}^{\prime}\left(t_{1}\right) d t_{1}
$$

and

$$
\frac{1}{b_{1}} \Delta_{\ell}\left(c, d, a_{1}, b_{1}\right) d s_{1}=f_{\ell}(c) d s-d f_{\ell}^{\prime}(t) d t .
$$

Let us start the computation of $d t_{2} \wedge d s_{2}$ using the last two identities. We obtain (using the rules of the exterior calculus)

$$
\begin{aligned}
d t_{2} \wedge d s_{2} & =\left(d t_{1}-\hat{c} d \tau_{1}\right) \wedge\left(d s_{1}-\frac{\hat{d}}{f_{\ell}(\hat{c})} d\left(f_{\ell}\left(t_{1}(t, s)\right)\right)\right) \\
& =-\hat{c} d \tau_{1} \wedge\left(d s_{1}-\frac{\hat{d}}{f_{\ell}(\hat{c})} d\left(f_{\ell}\left(t_{1}(t, s)\right)\right)\right) \\
& =\delta_{1} d \tau_{1} \wedge d s_{1}
\end{aligned}
$$

with

$$
\delta_{1}=-|\hat{c}|^{1-\ell} b_{1}^{-1} \Delta_{\ell}\left(\hat{c}, \hat{d}, a_{1}, b_{1}\right) .
$$

It remains to use the first relation of (6.9) which gives

$$
d \tau_{1}=\frac{1}{c} d t-\frac{1}{c} d t_{1}
$$

\footnotetext{
${ }^{4}$ See later in this paper for what we have to do when $\hat{c}=0$.
} 
and

$$
d t_{2} \wedge d s_{2}=\frac{\delta_{1}}{c} d t \wedge d s_{1}
$$

Using (6.12), we finally obtain the following lemma.

Lemma 6.1. If $\tau>\tau_{1}(t, s)$, then

$$
d t_{2} \wedge d s_{2}=\delta d t \wedge d s
$$

with

$$
\delta:=-|\hat{c}|^{1-\ell}|c|^{\ell-1} \frac{\Delta_{\ell}\left(\hat{c}, \hat{d}, a_{1}, b_{1}\right)}{\Delta_{\ell}\left(c, d, a_{1}, b_{1}\right)} .
$$

So the Jacobian is equal to $\delta$, hence constant, and nonzero. The fact that $\Delta_{\ell}\left(\hat{c}, \hat{d}, a_{1}, b_{1}\right)$ is not zero is the consequence of the assumption on the zeros of $\widetilde{\varphi}$.

Remark 6.2. The existence of this lower bound of the Jacobian is probably the key point. It is shown (see [JoTre]) in the analytic case that one can always find a $\gamma$ satisfying all the assumptions except this lower bound of the Jacobian by simply considering the flow associated with $\frac{1}{|\nabla \varphi|} \nabla \varphi$.

Now if we observe that $(-\varphi)$ has in $\mathcal{V}_{k}^{-}$the properties that $\varphi$ had in $\mathcal{V}_{1}^{+}$, we obtain rather easily the existence of $\mu>0$ such that

$$
\rho(\tau)^{m} \widetilde{\varphi}\left(\theta_{\tau}\right)-\rho^{m} \widetilde{\varphi}(\theta) \geq \mu \tau^{m}, \forall \tau \in\left[0, \tau_{1}(t, s)\right] .
$$

Here we can indeed use Lemma 5.1 (after exchange of the roles of $\theta_{\tau}$ and $\theta$ ).

We note also that, for $\tau>\tau_{1}(t, s)$, we are in a region where $\varphi$ is positive so that we can apply what we have done in this case. In particular, we obtain (see (4.10) ) with $\tau_{1}=\tau_{1}(t, s)$,

$$
\rho(\tau)^{m} \widetilde{\varphi}\left(\theta_{\tau}\right)-\rho\left(\tau_{1}\right) \widetilde{\varphi}\left(\theta_{\tau_{1}}\right) \geq \mu\left(\tau-\tau_{1}\right)^{m} .
$$

One can also observe (se65 (4.9)) that

$$
\rho(\tau)^{m}-\rho^{m} \leq-\left(\frac{\tau}{2}\right)^{m}, \forall \tau \in\left[0, \tau_{1}(t, s)\right],
$$

which implies in particular the upperbound

$$
\tau_{1}(t, s) \leq 2 \rho \leq \frac{1}{2}
$$

and the inequality (see again (4.9))

$$
\rho(\tau)^{m}-\rho\left(\tau_{1}\right)^{m} \geq\left(\frac{\tau-\tau_{1}}{2}\right)^{m}, \forall \tau \in\left[\tau_{1}, 1\right] .
$$

From (6.18) and (6.19), one obtains that

$$
\gamma(t, s, 1) \notin D\left(\frac{1}{4}\right) .
$$

So the escaping condition (i) of $\left(H_{+}(\alpha)\right)$ is satisfied with $\omega=D\left(\frac{1}{4}\right)$.

On the other hand, we get from (6.15) and (6.16), the estimate

$$
\rho(\tau)^{m} \widetilde{\varphi}\left(\theta_{\tau}\right)-\rho^{m} \widetilde{\varphi}(\theta) \geq 2^{-m} \mu \tau^{m}, \forall \tau \in[0,1] .
$$

\footnotetext{
${ }^{5}$ Here we use assumption 6.1.
} 
6.1.2. The subcase $c=0$. For definiteness, we assume that $c=0$ and $d=-1$, and look at initial data in the "sector" attached to the arc $\left(\left(a_{1}, b_{1}\right),(0,-1)\right)$. The point is just to choose a point $\left(c^{\prime}, d^{\prime}\right)$ in $\mathcal{S}$, with $c^{\prime}>0$ and sufficiently close to $(c, d)=(0,-1)$ in order to keep the condition

$$
\left\langle\left(a_{1}, b_{1}\right) \mid\left(c^{\prime}, d^{\prime}\right)\right\rangle>0 .
$$

We can then keep the previous construction with $(c, d)$ replaced by $\left(c^{\prime}, d^{\prime}\right)$.

6.2. Remaining case. In order to achieve the proof of Theorem 1.3 (i.e. to prove that $\left(H_{+}(\alpha)\right)$ is satisfied), we have to treat the case when (6.1) is no longer satisfied. So $(s, t) \mapsto\langle(c, d) \mid(t(\tau), s(\tau))\rangle$ may change of sign on $\mathcal{V}_{k}^{-}$. We have avoided this problem in the case of $\mathcal{V}_{k}^{+}$by dividing the "sector" in smaller "sectors", but this is no longer possible when $\widetilde{\varphi}$ is negative. In this general case, one will be obliged to add a broken line to the two previously defined arcs (ingoing and escaping) in order to leave $\omega$. We will see that it is always possible using a broken line made of at most five segments with the price of taking $\omega$ smaller.

We start from a point $(t, s)$ in the intersection of $\omega:=D(R)(R>0$ small enough) with a "sector" associated with the arc $\left(\left(a_{1}, b_{1}\right),(c, d)\right)$, and we divide this "sector" into $N$ "sectors" $S^{j}(j=1, \ldots, N)$ of disto-angle $<\frac{\pi}{2}$. They are delimited by "rays" attached to the sequence $\left(\widehat{\eta}_{j}, \widehat{\zeta}_{j}\right)(j=0, \ldots, N)$ in $\mathcal{S}$ going clockwise with $\left(\widehat{\eta}_{0}, \widehat{\zeta}_{0}\right)=(c, d),\left(\widehat{\eta}_{N}, \widehat{\zeta}_{N}\right)=\left(a_{1}, b_{1}\right)$.

For the commodity of the notation below, we also introduce $\left(\widehat{\eta}_{-1}, \widehat{\zeta}_{-1}\right)=\left(a_{2}, b_{2}\right)$ and $\left(\widehat{\eta}_{N+1}, \widehat{\zeta}_{N+1}\right)=\left(c_{0}, d_{0}\right)$.

It is clear that we can always do that with $N \leq 4$ and that we have previously treated the case $N=1$.

We also need another sequence $\left(\eta_{j}, \zeta_{j}\right)$ in $\mathcal{S}$, which will determine the various directions of the broken line and will satisfy

$$
\left\{\begin{array}{l}
\eta_{j} \neq 0 \\
\left(\eta_{j}, \zeta_{j}\right) \in\left(\left(\widehat{\eta}_{j-2}, \widehat{\zeta}_{j-2}\right),\left(\widehat{\eta}_{j-1}, \widehat{\zeta}_{j-1}\right)\right) \\
\Delta_{\ell}\left(\eta_{j}, \zeta_{j}, \widehat{\eta}_{j}, \widehat{\zeta}_{j}\right) \neq 0 \\
\Delta_{\ell}\left(\eta_{j+1}, \widehat{\zeta}_{j+1}, \widehat{\eta}_{j}, \widehat{\zeta}_{j}\right) \neq 0
\end{array}\right.
$$

This will be satisfied by taking $\left(\eta_{j}, \zeta_{j}\right)$ very close (but distinct except possibly for $N=1($ if $c \neq 0))$ to $\left(\widehat{\eta}_{j-1}, \widehat{\zeta}_{j-1}\right)$ for $j=1, \ldots, N$.

So we can always be assured by the property that starting from a point in the disk on $\mathcal{R}_{\widehat{\eta}_{j}, \widehat{\zeta}_{j}}$ the "straight" line parallel to $\left(\eta_{j+1}, \zeta_{j+1}\right)$ will meet the "ray" $\mathcal{R}_{\widehat{\eta}_{j+1}, \widehat{\zeta}_{j+1}}$ inside the "disk", for $j=1, \ldots, N-1$.

We now explain how we construct the broken line starting from a point $(t, s)$ belonging to the first "sector" $S_{1}:=S\left(\widehat{\eta}_{0}, \widehat{\zeta}_{0}, \widehat{\eta}_{1}, \widehat{\zeta}_{1}\right)$. The other cases are simpler.

Starting from $(t, s)$ we follow for $\tau \geq 0$ the parametrized "line" parallel to $\left(\eta_{1}, \zeta_{1}\right)$ until we meet at the time $\tau_{1}(t, s)$ the "ray" $\mathcal{R}_{\widehat{\eta}_{1}, \widehat{\zeta}_{1}}$ at the point $\left(t_{1}(t, s), s_{1}(t, s)\right)$.

If $N>1$ and starting now from $\left(t_{1}(t, s), t_{2}(t, s)\right)$, we follow for $\tau \geq \tau_{1}(t, s)$ the parametrized "line" parallel to $\left(\eta_{2}, \zeta_{2}\right)$ until we meet at the time $\tau_{2}(t, s)$ the "ray" $\mathcal{R}_{\widehat{\eta}_{2}, \widehat{\zeta}_{2}}$ at the point $\left(t_{2}(t, s), s_{2}(t, s)\right.$.

By recursion, we arrive at the point $\left(t_{N}(t, s), s_{N}(t, s)\right)$ on the "ray" $R_{\widehat{\eta}_{N}, \widehat{\zeta}_{N}}$ at time $\tau_{N}(t, s)$. We can then escape along $\left(\eta_{N+1}, \zeta_{N+1}\right)$. 
To generalize what we have done for $N=1$, we have now mainly to verify the following points:

(i) The condition of the Jacobians for $\tau \in] \tau_{j}(t, s), \tau_{j+1}(t, s)[$ :

This will be analyzed in the appendix,

(ii) The control of the escape time:

We have to determine a condition on $\omega$ such that

$$
\tau_{j+1}(t, s)-\tau_{j}(t, s) \leq \frac{1}{4 N} .
$$

(iii) The control of the dynamics:

We have to control $\rho\left(\tau_{j}(t, s)\right)$ for $j=1, \ldots, N$, under the suitable assumption that $(s, t) \in D\left(0, R_{N}\right)$.

(iv) The positivity of $\widetilde{\varphi}(\gamma(t, s, \tau))-\widetilde{\varphi}(t, s)$ along the trajectory:

We do not meet new problems here.

For the proof of (ii) and (iii) we observe that

$$
\left.\left.\rho(\tau)^{m}+\left(\frac{\tau-\tau_{i}}{2}\right)^{m} \leq \rho\left(\tau_{i}\right)^{m}, \forall \tau \in\right] \tau_{i}, \tau_{i+1}\right] .
$$

So in particular

$$
\rho\left(\tau_{i+1}\right)^{m}+\left(\frac{\tau_{i+1}-\tau_{i}}{2}\right)^{m} \leq \rho\left(\tau_{i}\right)^{m}
$$

This gives

$$
\rho\left(\tau_{i+1}\right) \leq \rho\left(\tau_{i}\right)
$$

and

$$
\tau_{i+1}-\tau_{i} \leq 2 \rho\left(\tau_{i}\right) \leq 2 \rho(0) .
$$

In addition, we have (see (6.19) for the case $N=1$ )

$$
\rho(\tau)^{m}-\rho\left(\tau_{N}(t, s)\right)^{m} \geq 2^{-m}\left(\tau-\tau_{N}(t, s)\right)^{m} .
$$

From this we deduce (without looking for optimality) that

$$
R_{N}=\frac{1}{8} \frac{1}{N}
$$

is enough to show that the parametrized broken line has left $\omega=D\left(0, R_{N}\right)$ at time $\tau=1$.

6.3. The case when $p>m$. Until now we have proved the main theorem under the additional condition (5.1). Without this additional condition, the subellipticity which is obtained is now $\inf \left(\frac{1}{m}, \frac{1}{p}\right)$ instead of $\frac{1}{m}$. The only change is that we get only the existence of $\mu>0$ such that, for $\tau \in[0,1]$,

$$
\rho(\tau)^{m} \varphi\left(\theta_{\tau}\right)-\rho^{m} \varphi(\theta) \geq \mu \inf \left(\tau^{m}, \rho(\tau)^{m}\left(\frac{\tau}{\rho(\tau)}\right)^{p}\right) .
$$

But we have shown the existence of a constant $C_{\varphi}>0$ and the existence of some open neighborhood of $0 \omega_{\varphi}$ such that

$$
0 \leq \tau \leq C_{\varphi} \rho(\tau) .
$$

So we get

$$
\rho(\tau)^{m} \varphi\left(\theta_{\tau}\right)-\rho^{m} \varphi(\theta) \geq \mu \tau^{\sup (m, p)} .
$$




\section{The Analytic CASE AND $\ell \in \mathbb{Q}$}

7.1. The main theorem in the analytic case. We keep the previous assumptions but now assume that

$$
\ell=\ell_{2} / \ell_{1}
$$

with $\ell_{1}$ and $\ell_{2}$ mutually prime integers, and that $\varphi$ is analytic. In this case, the quasihomogeneity assumption (1.7) on $\varphi$ implies that $\varphi$ is actually a polynomial and, we can write $\varphi$ in the form

$$
\varphi(t, s)=\sum_{\ell_{1} j+\ell_{2} k=\ell_{1} m} a_{j, k} t^{j} s^{k}
$$

where $(j, k)$ are integers and the $a_{j, k}$ are real.

We can of course apply the main theorem, but it is nicer to have a criterion involving more directly the assumptions on $\varphi$ or on its restriction $\hat{\varphi}$ of $\varphi$ to the quasicircle

$$
\mathcal{S}_{\ell_{1}, \ell_{2}}:=\left\{t^{2 \ell_{2}}+s^{2 \ell_{1}}=1\right\}
$$

instead of the disto-circle $\mathcal{S}$. There are absolutely no problems if the critical points or zeroes of $\widetilde{\varphi}$ avoid $\{t=0\} \cup\{s=0\}$, but one should be more careful in order to analyze condition (1.10) at the remaining points.

Let us show how this works in this case. We parametrize (this is a $C^{2}$ parametrization) $\mathcal{S}$ by $t$ and assume that we are close to $(0,1)$ for definiteness and $\widetilde{\varphi}$ becomes locally, with this parametrization, the function

$$
t \mapsto \kappa(t)=\varphi\left(t, \sqrt{1-t^{2 \ell}}\right)
$$

and we assume that $\kappa(0)=0$ and that $\kappa$ is not identically 0 . Suppose that we are on the side $\{t>0\}$. Then

$$
\kappa(t)=\chi\left(t^{\frac{1}{\ell_{1}}}\right),
$$

where $\chi$ is the nonidentically zero analytic function

$$
v \mapsto \chi(v)=\varphi\left(v^{\ell_{1}}, \sqrt{1-v^{2 \ell_{2}}}\right),
$$

with $\chi(0)=0$.

Now there exists $p>0$ such that $\chi^{(p)}(0) \neq 0$, and we get the existence of $C>0$ such that, in the neighborhood of 0 ,

$$
\forall v \geq 0, \forall v^{\prime} \geq 0,\left|\chi(v)-\chi\left(v^{\prime}\right)\right| \geq \frac{1}{C}\left|v-v^{\prime}\right|^{p} .
$$

Coming back to $\kappa$, we get for a constant $\widehat{C}>0$

$$
\forall t \geq 0, \forall t^{\prime} \geq 0,\left|\kappa(t)-\kappa\left(t^{\prime}\right)\right| \geq \frac{1}{C}\left|t^{\frac{1}{\ell_{1}}}-\left(t^{\prime}\right)^{\frac{1}{\ell_{1}}}\right|^{p} \geq \frac{1}{\widehat{C}}\left|t-t^{\prime}\right|^{p} .
$$

So we have obtained the proof of (1.10) for some $p$.

Theorem 7.1. Let $\varphi$ be a real analytic nonidentically 0 quasihomogeneous function satisfying (1.5), (1.6), and (1.7), with $\ell=\ell_{2} / \ell_{1}$. If $\varphi$ is strictly positive on $\mathbb{R}^{2} \backslash$ $\{0\}$, then $\varphi$ satisfies $\left(H_{+}(\alpha)\right)$ with $\alpha=\frac{\ell_{1}}{m}$ and the system (1.1) is $\frac{\ell_{1}}{m}$-microlocally subelliptic in $\{\xi>0\}$.

\footnotetext{
${ }^{6}$ Using the fact that $\varphi$ is a polynomial, one could get more information on $p$ if needed.
} 
Theorem 7.2. Let $\varphi$ be a real analytic nonidentically 0 quasihomogeneous function satisfying (1.5), (1.6), and (1.7), with $\ell=\ell_{2} / \ell_{1}$. Suppose that $\varphi$ is not a negative function. Suppose in addition that:

If $\mathcal{S}_{k}^{-}=\left[\theta_{k}, \theta_{k+1}\right]$ is a maximal arc where $\widehat{\varphi}$ is negative, then $\widehat{\varphi}^{\prime}$ has a unique zero on $] \theta_{k}, \theta_{k+1}[$.

Then $\varphi$ satisfies $\left(H_{+}(\alpha)\right)$ with $\alpha>0$. Hence the system (1.1) is microlocally subelliptic in $\{\xi>0\}$.

Example 7.3. We recover some examples treated by H. Maire [Mai4]:

$$
\varphi(t, s)=t\left(s^{2}-t^{2 \ell}\right), \ell \geq 1 .
$$

Here $m=2 \ell+1, p=1$ and we get the subellipticity with $\alpha=\frac{1}{2 \ell+1}$. As observed in $[\mathrm{HeNi}$, this result is optimal and the associated system is not maximally hypoelliptic when $\ell>1$ (see Appendix B for a more elaborate discussion). The maximal hypoellipticity would indeed imply $\alpha=\frac{1}{3}$.

\subsection{On Journé-Trépreau's examples. For}

$$
\varphi(t, s)=-t^{2 m}-t^{2} s^{2 p}+s^{q}
$$

with

$$
m \geq 1, p \geq 2, q \geq \frac{2 m p}{m-1},
$$

J.L. Journé and J.M. Trépreau show that, although the Maire-Trèves condition is satisfied, one cannot obtain a better $\rho$-subellipticity than

$$
\rho \leq-\left(1-\frac{2 p}{q}-\frac{1}{m}\right) \frac{n-1}{4}+\frac{1}{2 q}+\frac{m-1}{4 m p} .
$$

The right hand side can become strictly negative. But if we impose the quasihomogeneity condition (17.2), we get as a necessary condition

$$
\left(1-\frac{2 p}{q}-\frac{1}{m}\right)=0
$$

which cancels the only possible negative term.

Indeed inside this class $(m=2, p=2)$, the authors can obtain the optimal subellipticity for the example

$$
\varphi(t, s)=-t^{4}-t^{2} s^{4}+s^{q},
$$

with $q \geq 8$.

The optimal subellipticity is $\rho_{q}=\frac{3}{2 q}-\frac{1}{16}$. Here let us observe that the only quasihomogeneous case corresponds to $q=8$ and that in this case their result is coherent with our result. This example also shows that we lose the "positive" subellipticity for $q \geq 24$.

7.3. Final remarks. In the analytic case, the criterion of microhypoellipticity (proven by Maire) at say $(0,0)$ in the direction $\{\xi>0\}$ is that $\varphi$ has no local maximum in a neighborhood of 0 . When $\varphi$ is quasihomogeneous, we immediately see that, at a local maximum, $\widetilde{\varphi}$ should be zero. So we should avoid the following situations:

- $\widetilde{\varphi} \leq 0$, with $\widetilde{\varphi}^{-1}(]-\infty, 0[) \neq \emptyset$.

- $\widetilde{\varphi}$ has a local maximum equal to zero on $\mathcal{S}$. 
One can indeed verify that our assumptions exclude these two cases but are unfortunately more restrictive.

Note also that it would be interesting to understand the case when $\widetilde{\varphi}$ or $\widehat{\varphi}$ have a strictly negative local maximum. We have not been able by our method to construct escaping curves satisfying all the conditions of the criterion in this case.

Finally, let us recall that the maximal hypoellipticity of these systems was analyzed in [HeNo, No1, No2, No3] and more recently in [HeNi]. We give in a second appendix some examples of applications of these techniques.

\section{Appendix A. Control of the Jacobian for an $(N+1)$-Broken line}

We just adapt the proof done for a broken line with two pieces. We treat for definiteness the case $N=2$ and keep the same notation as in the main text.

Starting from $(t, s)$ we consider the parametrized (disto)-line

$$
t_{1}(\tau, t, s)=t-\eta_{1} \tau, s_{1}(\tau, t, s)=s+\frac{\zeta_{1}}{f_{\ell}\left(\eta_{1}\right)}\left(f_{\ell}\left(t_{1}(\tau, t, s)\right)-f_{\ell}(t)\right) .
$$

Here $\left(\eta_{1}, \zeta_{1}\right)$ is a point of $\mathcal{S}$ with $\eta_{1} \neq 0$ and, in our example (if in addition $c \neq 0$ ), $\left(\eta_{1}, \zeta_{1}\right)=(c, d)$.

We refer to (6.22) for the conditions on the sequence $\left(\eta_{j}, \zeta_{j}\right)$ and $\left(\widehat{\eta}_{j}, \widehat{\zeta}_{j}\right)$. We simply add the equations which are satisfied at the singular points of the broken line. The intersection with the "ray" $\mathcal{R}_{\widehat{\eta}_{1}, \widehat{\zeta}_{1}}$ occurs at time $\tau_{1}(t, s)$, and we get the first family of relations for the intersection point denoted by $\left(t_{1}(t, s), s_{1}(t, s)\right)$ :

$$
\begin{aligned}
& \Delta_{\ell}\left(\widehat{\eta}_{1}, \widehat{\zeta}_{1}, t_{1}(t, s), s_{1}(t, s)\right)=0 ; \\
& \Delta_{\ell}\left(\eta_{1}, \zeta_{1}, t_{1}(t, s), s_{1}(t, s)\right)=\Delta_{\ell}\left(\eta_{1}, \zeta_{1}, t, s\right) ; \\
& t_{1}(t, s)=t-\eta_{1} \tau_{1}(t, s) .
\end{aligned}
$$

Starting from $\left(t_{1}(t, s), s_{1}(t, s)\right)$ we construct a new ingoing "straight"' 7 half-line $\mathcal{L}_{2}(t, s)$ disto-parallel to the disto-unit-vector $\left(\eta_{2}, \zeta_{2}\right)$.

The straight line meets $\mathcal{R}_{\widehat{\eta}_{2}, \widehat{\zeta}_{2}}$ at time $\tau_{2}(t, s)$ and at the point $\left(t_{2}(t, s), s_{2}(t, s)\right)$. So we get the second family of relations:

$$
\begin{aligned}
& \Delta_{\ell}\left(\widehat{\eta}_{2}, \widehat{\zeta}_{2}, t_{2}(t, s), s_{2}(t, s)\right)=0 ; \\
& \Delta_{\ell}\left(\eta_{2}, \zeta_{2}, t_{2}(t, s), s_{2}(t, s)\right)=\Delta_{\ell}\left(\eta_{2}, \zeta_{2}, t_{1}(t, s), s_{1}(t, s)\right) ; \\
& t_{2}(t, s)=t_{1}(t, s)-\eta_{2}\left(\tau_{2}(t, s)-\tau_{1}(t, s)\right) .
\end{aligned}
$$

For $\tau>\tau_{2}(t, s)$, we now consider the last half-line, which is now assumed to be escaping and starting from $\left(t_{2}(t, s), s_{2}(t, s)\right)$, and parallel to a new vector $\left(\eta_{3}, \zeta_{3}\right)$. At time $\tau$, we will be at the point

$$
\begin{aligned}
& t_{3}(\tau, t, s)=t_{2}(t, s)+\eta_{3}\left(\tau-\tau_{2}(t, s)\right), \\
& s_{3}(\tau, t, s)=s_{2}(t, s)+\frac{\zeta_{3}}{f_{\ell}\left(\eta_{3}\right)}\left(f_{\ell}\left(t_{3}(\tau, t, s)\right)-f_{\ell}\left(t_{2}\right)\right) .
\end{aligned}
$$

It remains to control the Jacobian for the different values of $\tau \in[0,1]$, which can be done by the computation of the coefficient of the 2 -form $d t_{3} \wedge d s_{3}$ on the 2 -form $d t \wedge d s$. We will see that this coefficient is locally constant.

The proof is similar to the proof of Lemma 6.1

We are actually able to give explicit formulas for these Jacobians, once the two sequences $\left(\eta_{j}, \zeta_{j}\right)$ and $\left(\widehat{\eta}_{j}, \widehat{\zeta}_{j}\right)$ are fixed.

\footnotetext{
${ }^{7}$ More precisely, straight after application of the dressing map.
} 
Let us treat for definiteness the case $N=2$ and look first at what is going on for $\tau \geq \tau_{2}(t, s)$.

We will show

$$
d t_{3} \wedge d s_{3}=\underline{\delta}_{32} d \tau_{2} \wedge d s_{2}=\underline{\delta}_{32} \widehat{\delta}_{21} d \tau_{1} \wedge d s_{1}=\delta_{3,0} d t \wedge d s .
$$

Let us show the existence of $\underline{\delta}_{3}$. Using (A.3), we first get

$$
d t_{3} \wedge d s_{3}=d t_{3} \wedge\left(d s_{2}-\frac{\zeta_{3}}{f_{\ell}\left(\eta_{3}\right)} f_{\ell}^{\prime}\left(t_{2}\right) d t_{2}\right) .
$$

Now, the first line of $($ A.2 gives us that

$$
f_{\ell}\left(\widehat{\eta}_{2}\right) d s_{2}-\widehat{\zeta}_{2} f_{\ell}^{\prime}\left(t_{2}\right) d t_{2}=0 .
$$

We then easily obtain

$$
\underline{\delta}_{32}=-\eta_{3}\left(1-\frac{f_{\ell}\left(\widehat{\eta}_{2}\right) \zeta_{3}}{f_{\ell}\left(\eta_{3}\right) \widehat{\zeta}_{2}}\right)=-\left|\eta_{3}\right|^{1-\ell} \widehat{\zeta}_{2}^{-1} \Delta_{\ell}\left(\eta_{3}, \zeta_{3}, \widehat{\eta}_{2}, \widehat{\zeta}_{2}\right)
$$

which should be nonzero in our construction.

The second step is to show that

$$
d \tau_{2} \wedge d s_{2}=\widehat{\delta}_{21} d \tau_{1} \wedge d s_{1}
$$

The differentiation of the third line of (A.2) gives

$$
d \tau_{2} \wedge d s_{2}=\left(\frac{1}{\eta_{2}} d t_{1}+d \tau_{1}\right) \wedge d s_{2} .
$$

The differentiation of the second line of (A.2) (together with (A.6)) permits us to express $d s_{2}$ as a combination of $d s_{1}$ and $f_{\ell}^{\prime}\left(t_{1}\right) d t_{1}$.

We indeed get

$$
d s_{2}=\frac{\widehat{\zeta}_{2}}{\Delta_{\ell}\left(\eta_{2}, \zeta_{2}, \widehat{\eta}_{2}, \widehat{\zeta}_{2}\right)}\left(f_{\ell}\left(\eta_{2}\right) d s_{1}-\zeta_{2} f_{\ell}^{\prime}\left(t_{1}\right) d t_{1}\right),
$$

and by differentiating the first line of (A.1), we get

$$
\widehat{\delta}_{21}=\frac{\widehat{\zeta}_{2}}{\Delta_{\ell}\left(\eta_{2}, \zeta_{2}, \widehat{\eta}_{2}, \widehat{\zeta}_{2}\right)} \frac{\Delta_{\ell}\left(\eta_{2}, \zeta_{2}, \widehat{\eta}_{1}, \widehat{\zeta}_{1}\right)}{\widehat{\zeta}_{1}} .
$$

The last step is to show

$$
d \tau_{1} \wedge d s_{1}=\bar{\delta}_{10} d t \wedge d s .
$$

The differentiation of the first line of (A.1) leads to

$$
d \tau_{1} \wedge d s_{1}=\frac{1}{\eta_{1}} d t \wedge d s_{1} .
$$

It then remains to use the two first lines of (A.1), and we obtain

$$
\bar{\delta}_{1,0}=\left|\eta_{1}\right|^{\ell-1} \frac{\widehat{\zeta}_{1}}{\Delta_{\ell}\left(\eta_{1}, \zeta_{1}, \widehat{\eta}_{1}, \widehat{\zeta}_{1}\right)} .
$$

So finally, we have obtained, observing that

$$
\begin{gathered}
\delta_{3,0}=\underline{\delta}_{3,2} \widehat{\delta}_{2,1} \bar{\delta}_{1,0} \\
\delta_{3,0}=-\left|\eta_{3}\right|^{1-\ell}\left|\eta_{1}\right|^{\ell-1} \frac{\Delta_{\ell}\left(\eta_{3}, \zeta_{3}, \widehat{\eta}_{2}, \widehat{\zeta}_{2}\right)}{\Delta_{\ell}\left(\eta_{2}, \zeta_{2}, \widehat{\eta}_{2}, \widehat{\zeta}_{2}\right)} \frac{\Delta_{\ell}\left(\eta_{2}, \zeta_{2}, \widehat{\eta}_{1}, \widehat{\zeta}_{1}\right)}{\Delta_{\ell}\left(\eta_{1}, \zeta_{1}, \widehat{\eta}_{1}, \widehat{\zeta}_{1}\right)} .
\end{gathered}
$$


Let us recapitulate what we have obtained in the case of the three-broken line.

(i) For $\tau \in\left[0, \tau_{1}(t, s)[\right.$, the Jacobian is one:

$$
\delta^{(1)}=\delta_{1,0}=1
$$

(ii) For $\tau \in] \tau_{1}(t, s), \tau_{2}(t, s)[$, the Jacobian (see the main text) is

$$
\delta^{(2)}:=\delta_{2,0}=-\left|\eta_{2}\right|^{1-\ell}\left|\eta_{1}\right|^{\ell-1} \frac{\Delta_{\ell}\left(\eta_{2}, \zeta_{2}, \widehat{\eta}_{1}, \widehat{\zeta}_{1}\right)}{\Delta_{\ell}\left(\eta_{1}, \zeta_{1}, \widehat{\eta}_{1}, \widehat{\zeta}_{1}\right)}
$$

(iii) For $\tau \in] \tau_{2}(t, s),+\infty\left[\right.$, the Jacobian is given by $\delta^{(3)}=\delta_{3,0}$.

Generalization. It is now not too difficult to extend the formula in the case of $N$ reflexions:

$$
\delta^{(N+1)}=-\left|\eta_{N+1}\right|^{1-\ell}\left|\eta_{1}\right|^{\ell-1} \Pi_{j=1}^{N}\left(\frac{\Delta_{\ell}\left(\eta_{j+1}, \zeta_{j+1}, \widehat{\eta}_{j}, \widehat{\zeta}_{j}\right)}{\Delta_{\ell}\left(\eta_{j}, \zeta_{j}, \widehat{\eta}_{j}, \widehat{\zeta}_{j}\right)}\right)
$$

\section{APPEndix B. On MAXIMAL hyPOELLIPTICITY}

We would like to analyze if the considered models are actually maximally hypoelliptic (in the sense of Helffer-Nourrigat [HeNo]). Maximal hypoellipticity simply means here the existence, for any relatively compact open sets $\omega, I$, of a constant $C(\omega, I)$ such that

$$
\sum_{j}\left\|X_{j} u\right\|^{2}+\left\|Y_{j} u\right\|^{2} \leq C \sum_{j}\left\|L_{j} u\right\|^{2}, \forall u \in C_{0}^{\infty}(\omega \times I),
$$

with $X_{j}=\frac{\partial}{\partial t_{j}}$ and $Y_{j}=\frac{\partial \varphi}{\partial t_{j}} \frac{\partial}{\partial x}$.

It has been proved in [HeNi] that Maire's model (7.3) is not maximally hypoelliptic as soon it is not homogeneous (the case $\ell>1$ ).

On the contrary, when $\ell=1$, i.e. if we look at

$$
\varphi\left(x_{1}, x_{2}\right)=x_{1}^{3}-x_{1} x_{2}^{2},
$$

one can show that the corresponding system is maximally hypoelliptic.

One can indeed apply Helffer-Nourrigat's criterion (se 8 also Nourrigat [No1, Helffer-Nier [HeNi] and the recent course by Nier $[\mathrm{Ni}$ ). We are in the case $r=3$. We can, in the verification of the criterion, distinguish between the representations associated to $E_{r}$ between:

(i) the nondegenerate representations, for which the criterion is implied directly by Maire's condition of hypoellipticity,

(ii) and the degenerate representations, for which one can proceed as in Subsection 10.4.2 in [HeNi].

For the second item, we indeed observe that

$$
|\nabla \varphi|^{2}=\left(3 x_{1}^{2}-x_{2}^{2}\right)^{2}+4 x_{1}^{2} x_{2}^{2}
$$

is nonzero for $x \neq(0,0)$.

It has then been shown in $\mathrm{HeNi}$ that the (nontrivial) limiting polynomials constructed from $\varphi$ (permitting us to determine the involved degenerate representations) cannot have any local minimum or maximum, because these polynomials are of order 1 and vanishing at the origin.

\footnotetext{
${ }^{8}$ Theorem 2 (pp. 134-135) in [No1] and Propositions 10.11 and 10.12 (p. 104) in [HeNi].
} 
One has consequently shown in this model that a criterion for maximal hypoellipticity in the homogeneous case is for example the criterion of hypoellipticity with in addition the condition that $|\nabla \varphi| \neq 0$ for $x \neq 0$.

In the two dimensional case, this corresponds, when restricting $\varphi$ to the unique disk, to the condition that this restriction of $\widetilde{\varphi}$ changes sign (with zero of order 1) at each of its zeros.

This suggests the conjecture that microlocal hypoellipticity (in the +-direction) is equivalent to the maximal microlocal hypoellipticity (in the +-direction) when $\varphi$ is a homogeneous polynomial.

\section{ACKNOWLEDGEMENTS}

At various stages of this work, the authors have had fruitful discussions with F. Nier, J.M. Trépreau and H. Maire. Some of these results were discussed or presented during stays of the authors at Erwin Schrödinger Institut. The authors would like to thank F. Haslinger for his invitation.

\section{REFERENCES}

[De] M. Derridj. Subelliptic estimates for some systems of complex vector fields. In "Hyperbolic problems and regularity questions". Series Trends in Mathematics. Eds.: M. Padula and L. Zanghirati. Birkhäuser (2006), p. 101-108. MR2298786 (2007k:35058)

[HeNi] B. Helffer and F. Nier. Hypoelliptic estimates and spectral theory for Fokker-Planck operators and Witten Laplacians. Lecture Notes in Math. 1862, Springer-Verlag, Berlin 2005. MR2130405 (2006a:58039)

[HeNo] B. Helffer and J. Nourrigat. Hypoellipticité maximale pour des opérateurs polynômes de champs de vecteur. Progress in Mathematics, Birkhäuser, Vol. 58 (1985). MR 897103 (88i:35029)

[Ho1] L. Hörmander. Hypoelliptic second order differential equations. Acta Mathematica 119 (1967), p. 147-171. MR0222474 (36:5526)

[Ho2] L. Hörmander. Subelliptic operators. Seminar on singularities of solutions of partial differential equations. Ann. Math. Studies 91 (1978), p. 127-208. MR.547019 (82e:35029)

[JoTre] J.L. Journé and J.M. Trépreau. Hypoellipticité sans sous-ellipticité: le cas des systèmes de $n$ champs de vecteurs complexes en $(n+1)$ - variables. Séminaire EDP in Ecole Polytechnique, April 2006. MR2276079(2007k:35055)

[Ko1] J. Kohn. Lectures on degenerate elliptic problems. Pseudodifferential operators with applications, C.I.M.E., Bressanone 1977 (1978), p. 89-151. MR660652 (84a:35059)

[Ko2] J.J. Kohn. Hypoellipticity and loss of derivatives, with an appendix by M. Derridj and D. Tartakoff. Ann. of Math. 162(2) (2005), p. 943-986. MR2183286 (2006k:35036)

[Mai1] H.M. Maire. Hypoelliptic overdetermined systems of partial differential equations. Comm. Partial Differential Equations 5 (4) (1980), p. 331-380. MR567778 (81f:35027)

[Mai2] H.M. Maire. Résolubilité et hypoellipticité de systèmes surdéterminés. Séminaire Goulaouic-Schwartz 1979-1980, Exp. V, Ecole Polytechnique (1980). MR600689 (83k:35026)

[Mai3] H.M. Maire. Necessary and sufficient conditions for maximal hypoellipticity of $\bar{\partial}_{b}$. Unpublished (1979).

[Mai4] H.M. Maire. Régularité optimale des solutions de systèmes différentiels et du Laplacien associé : application au $\square_{b}$. Math. Ann. 258 (1981), p. 55-63. MR641668 (83c:35027)

[Ni] F. Nier. Hypoellipticity for Fokker-Planck operators and Witten Laplacians. Course in China. Preprint September 2006.

[No1] J. Nourrigat. Subelliptic estimates for systems of pseudo-differential operators. Course in Recife (1982). University of Recife.

[No2] J. Nourrigat. Systèmes sous-elliptiques. Séminaire Equations aux Dérivées Partielles, 19861987, exposé V, Ecole Polytechnique (1987). MR920023 (89d:35034)

[No3] J. Nourrigat. Subelliptic systems II. Inv. Math. 104 (2) (1991), p. 377-400. MR1098615 (92f:35048) 
[Tr1] F. Trèves. A new method of proof of the subelliptic estimates. Comm. Pure Appl. Math. 24 (1971), p. 71-115. MR0290201 (44:7385)

[Tr2] F. Trèves. Study of a model in the theory of complexes of pseudo-differential operators. Ann. of Math. (2) 104 (1976), p. 269-324. See also erratum: Ann. of Math. (2) 113 (1981), p. 423. MR0426068 (54:14014) MR0607900 (82i:35044)

5 Rue de la Juvinière, 78350 Les Loges en Josas, France

Laboratoire de Mathématiques, Univ Paris-Sud and CNRS, F 91405 Orsay Cedex, FRANCE 